\title{
Efficient and Privacy-Aware Data Aggregation in Mobile Sensing
}

\author{
Qinghua Li, Guohong Cao, Thomas F. La Porta \\ Department of Computer Science and Engineering \\ The Pennsylvania State University \\ Email: $\{$ qx1118, gcao, tlp $\} @$ cse.psu.edu
}

\begin{abstract}
The proliferation and ever-increasing capabilities of mobile devices such as smart phones give rise to a variety of mobile sensing applications. This paper studies how an untrusted aggregator in mobile sensing can periodically obtain desired statistics over the data contributed by multiple mobile users, without compromising the privacy of each user. Although there are some existing works in this area, they either require bidirectional communications between the aggregator and mobile users in every aggregation period, or have high computation overhead and cannot support large plaintext spaces. Also, they do not consider the Min aggregate which is quite useful in mobile sensing. To address these problems, we propose an efficient protocol to obtain the Sum aggregate, which employs an additive homomorphic encryption and a novel key management technique to support large plaintext space. We also extend the sum aggregation protocol to obtain the Min aggregate of time-series data. To deal with dynamic joins and leaves of mobile users, we propose a scheme which utilizes the redundancy in security to reduce the communication cost for each join and leave. Evaluations show that our protocols are orders of magnitude faster than existing solutions, and it has much lower communication overhead.
\end{abstract}

Index Terms-Mobile sensing, privacy, data aggregation

\section{INTRODUCTION}

Mobile devices such as smart phones are gaining an everincreasing popularity. Most smart phones are equipped with a rich set of embedded sensors such as camera, microphone, GPS, accelerometer, ambient light sensor, gyroscope, etc. The data generated by these sensors provides opportunities to make sophisticated inferences about not only people (e.g., human activity, health, location, social event) but also their surrounding (e.g., pollution, noise, weather, oxygen level), and thus can help improve people's health as well as life. This enables various mobile sensing applications such as environmental monitoring [1], traffic monitoring [2], healthcare [3], etc.

In many scenarios, aggregation statistics need to be periodically computed from a stream of data contributed by mobile users [4], in order to identify some phenomena or track some important patterns. For example, the average amount of daily exercise (which can be measured by motion sensors [5]) that people do can be used to infer public health conditions. The average or maximum level of air pollution and pollen concentration in an area may be useful for people to plan their outdoor activities. Other statistics of interests include the lowest gasoline price in a city, the highest moving speed of road traffic during rush hour, etc.

Although aggregation statistics computed from time-series data are very useful, in many scenarios, the data from users is privacy-sensitive, and users do not trust any single third-party aggregator to see their data values. For instance, to monitor the propagation of a new flu, the aggregator will count the number of users infected by this flu. However, a user may not want to directly provide her true status (" 1 " if being infected and " 0 " otherwise) if she is not sure whether the information will be abused by the aggregator. Accordingly, systems that collect users' true data values and compute aggregate statistics over them may not meet users' privacy requirement [4]. Thus, an important challenge is how to protect the users' privacy in mobile sensing, especially when the aggregator is untrusted.

Most previous works on sensor data aggregation assume a trusted aggregator, and hence cannot protect user privacy against an untrusted aggregator in mobile sensing applications. Several recent works [6]-[9] consider the aggregation of timeseries data in the presence of an untrusted aggregator. To protect user privacy, they design encryption schemes in which the aggregator can only decrypt the sum of all users' data but nothing else. Rastogi and Nath [6] use threshold Paillier cryptosystem [10] to build such an encryption scheme. To decrypt the sum, their scheme needs an extra round of interaction between the aggregator and all users in every aggregation period, which means high communication cost and long delay. Moreover, it requires all users to be online until decryption is completed, which may not be practical in many mobile sensing scenarios due to user mobility and the heterogeneity of user connectivity. Rieffel et al. [9] propose a construction that does not require bidirectional communications between the aggregator and the users, but it has high computation and storage cost to deal with collusions in a large system.

Shi et al. [7], [8] also propose a construction for sum aggregation which does not need the extra round of interaction. However, the decryption in their construction needs to traverse the possible plaintext space of the aggregated value, which is very expensive for a large system with large plaintext space. In mobile sensing, the plaintext space of some application can be large. For example, carbon dioxide levels can range from $350 \mathrm{ppm}$ outdoors to over $10000 \mathrm{ppm}$ in industrial workplaces [11]. Hence in applications which continuously monitor the carbon dioxide levels that people are exposed to in their daily life [12], [13], the plaintext space can reach $10^{4}$. Under this plaintext space, for a large system with one million users, the construction in [7] requires 30 seconds to decrypt the sum 
on a modern 64-bit desktop PC. Its computation overhead is too high for an aggregator to run realtime monitoring applications with short aggregation intervals and to collect multiple aggregate statistics simultaneously. Moreover, none of these existing schemes considers the Min aggregate (i.e., the minimum value) of time-series data, which is also important in many mobile sensing applications.

In this paper, we propose a new protocol for mobile sensing to obtain the sum aggregate of time-series data in the presence of an untrusted aggregator. Our protocol employs an additive homomorphic encryption and a novel key management scheme based on efficient HMAC to ensure that the aggregator can only obtain the sum of all users' data, without knowing individual user's data or intermediate result. In our protocol, each user (the aggregator) only needs to compute a very small number of HMACs to encrypt her data (decrypt the sum). Hence, the computation cost is very low, and the protocol can scale to large systems with large plaintext spaces, resourceconstrained devices and high aggregation loads. Another nice property of our protocol is that it only requires a single round of user-to-aggregator communication.

Based on the sum aggregation protocol, we propose a protocol to obtain the Min aggregate. To our best knowledge, this is the first privacy-preserving solution to obtain the Min of time-series data in mobile sensing with just one round of userto-aggregator communication. Our protocols for Sum and Min can be easily adapted to derive many other aggregate statistics such as Count, Average and Max.

Since users may frequently join and leave in mobile sensing, we also propose a scheme which employs the redundancy in security to reduce the communication cost of dealing with dynamic joins and leaves.

The remainder of this paper is organized as follows. Section II discusses related work. Section III presents system models and assumptions. Section IV and Section V present our protocols for Sum and Min, respectively. Section VI presents our scheme to deal with dynamic joins and leaves. Section VII evaluates the practical performance and cost of our solutions. The last two sections present discussions and conclusions.

\section{RELATED WORK}

Many works have addressed various security and privacy issues in mobile sensing networks and systems (e.g., [14][18]), but they do not consider data aggregation. There are a lot of existing works (e.g., [19]-[21]) on security and privacypreserving data aggregation, but most of them assume a trusted aggregator and cannot protect user privacy against untrusted aggregators. Yang et al. [22] proposed an encryption scheme that allows an untrusted aggregator to obtain the sum of multiple users's data without knowing any specific user's data. However, their scheme requires expensive re-keying operations to support multiple time steps, and thus may not work for time-series data.

Shi et al. [23] proposed a privacy-preserving data aggregation scheme based on data slicing and mixing techniques. However, their scheme is not designed for time-series data.
It may not work well for time-series data, since each user may need to select a new set of peers in each aggregation interval due to mobility. Besides, their scheme for non-additive aggregates (e.g., Max/Min) requires multiple rounds of bidirectional communications between the aggregator and mobile users which means long delays. In contrast, our scheme obtains those aggregates with just one round of uni-directional communication from users to the aggregator.

To achieve privacy-preserving sum aggregation of timeseries data, Rastogi and Nath [6] designed an encryption scheme based on threshold Paillier cryptosystem [10], where the decryption key is divided into portions and distributed to the users. The aggregator collects the ciphertexts of users, multiplies them together and sends the aggregate ciphertext to all users. Each user decrypts a share of the sum aggregate. The aggregator collects all the shares and gets the final sum. However, their scheme requires an extra round of interaction between the aggregator and users in every aggregation period. Erkin and Tsudik [24] also proposed an aggregation scheme based on Paillier cryptosystem, but it requires communications between every pair of users in every aggregation period.

Based on an efficient additive homomorphic encryption scheme, Rieffel et al. [9] proposed a construction that does not require an extra round of interaction between the aggregator and the users. In their scheme, the computation and storage cost is roughly equal to the number of colluding users that the system can tolerate. Thus, their scheme has high overhead to achieve good resistance to collusion, especially when the system is large and a large number of users collude. In contrast, our scheme tolerates a high fraction of colluding users (e.g., 30\%) with very small cost even when the system is large. Acs and Castelluccia [25] also proposed a scheme based on additive homomorphic encryption, but in their scheme each node shares a pairwise key with any other node.

Shi et al. [7] proposed a construction for sum aggregation based on the assumption that the Decisional Diffie-Hellman problem is hard over finite cyclic groups. In their construction, each user sends her ciphertext to the aggregator and no communication is needed from the aggregator to the users. To decrypt the sum, their construction needs to traverse the possible plaintext space of sum, and thus it is not efficient for a large system with large plaintext spaces. Chan et al. [8] extended the construction in [7] with a binary interval tree technique, but their scheme still has the limitation in plaintext spaces. Jawurek and Kerschbaum [26] proposed a scheme which provides differential privacy for sum. Our aggregation protocol for sum can be used as a building block of their scheme to improve the computational efficiency. Also, those works [6]-[9], [24]-[27] do not consider the Min of time-series data.

\section{PRELIMINARIES}

\section{A. Models and Assumptions}

Figure 1 shows our system model, which is similar to the model in [7]. An aggregator wishes to get the aggregate statistics of $n$ mobile users periodically, e.g., in every hour. 
The time periods are numbered as $1,2,3, \ldots$, etc. In every time period, each user $i$ encrypts her data $x_{i}$ with key $k_{i}$ and sends the derived ciphertext to the aggregator. From the ciphertexts, the aggregator decrypts the needed aggregate statistics using her aggregator capability $k_{0}$. The value of each user's data is an integer within range $[0, \Delta]$. Two types of aggregate statistics are considered in this work, which are Sum and Min. Sum is defined as the sum of all users' data and Min is defined as the minimum value of the users' data. From Sum and Min, many other aggregate statistics can be easily derived, such as Count (i.e., the number of users that satisfy certain predicate), Average (which is derivable from Sum and Count), and Max (which can be obtained from the Min of $\Delta-x$ ).

In each time period, a mobile user sends her encrypted data to the aggregator via $\mathrm{WiFi}, 3 \mathrm{G}$ or other available access networks. No peer-to-peer communication is required among mobile users, since such communication is nontrivial in mobile sensing scenarios due to the high mobility of users and users may not be aware of each other for privacy reasons.

We consider an untrusted aggregator that is curious about each individual user's data. The aggregator may eavesdrop all the messages sent from/to every user. A number of users may collude with the aggregator, and reveal their data to the aggregator. A number of users may also collude to obtain the aggregate. Similar to [7], we assume that the fraction of users that collude is at most $\gamma(0 \leq \gamma<1)$, and the system has a priori estimate over the upper bound of $\gamma$ which can be used in practice. For now, we assume that $\gamma$ is the maximum accumulated fraction of users that collude during the lifetime of the system, and we relax this assumption in Section VIII. In addition, the aggregator and users have limited computation capability. Note that this paper focuses on thwarting attacks against users' privacy. Other important issues such as data pollution attacks (in which malicious users provide false data values to sway the final aggregate statistics) are not considered.

We assume a key dealer which issues proper keys to the aggregator and users via a secure channel. For now, the key dealer is assumed to be trusted, and this assumption is relaxed in Section VIII.

Our goal is to guarantee the privacy of each user's data against the untrusted aggregator, i.e., the aggregator obtains the aggregate statistics without knowing any individual user's data. We achieve this goal through protecting each user's data content with an encryption scheme, but not through providing source anonymity [28]. Also, we guarantee that any party without an appropriate aggregator capability obtains nothing.

\section{B. Underlying Encryption Scheme}

One building block of our solution is the additive homomorphic encryption scheme proposed by Castelluccia et al. [20], [29]. This scheme works as follows.

Encryption:

1) Represent message $m$ as an integer within range [0, $M-$ 1] where $M$ is a large integer.

2) Let $k$ be a randomly generated key, $k \in\{0,1\}^{\lambda}$, where $\lambda$ is a security parameter.

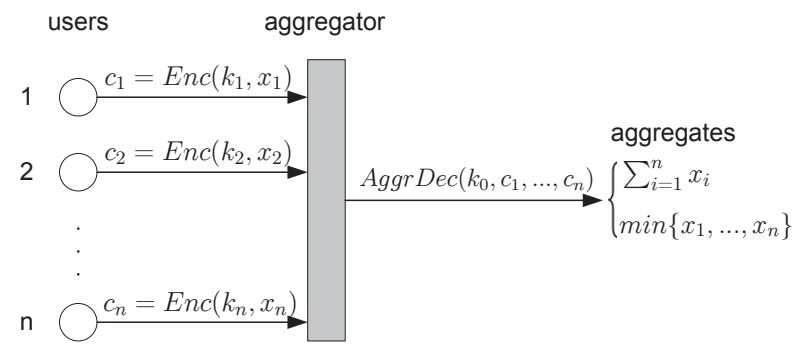

Fig. 1. Our system model of time-series data aggregation.

3) Output ciphertext $c=\left(m+h\left(f_{k}(r)\right)\right) \bmod M$, where $f_{k}$ is a pseudorandom function (PRF) that uses $k$ as a parameter, $h$ is a length-matching hash function (see details below) and $r$ is a nonce for this message.

\section{Decryption:}

1) Output plaintext $m=\left(c-h\left(f_{k}(r)\right)\right) \bmod M$.

The PRF $f_{k}$ is a function of the PRF family $\mathbb{F}_{\lambda}=\left\{f_{k}\right.$ : $\left.\{0,1\}^{\lambda} \rightarrow\{0,1\}^{\lambda}\right\}_{k \in\{0,1\}^{\lambda}}$ indexed by $k$. Since provably secure PRFs are usually computationally expensive, Castelluccia et al. [20] advocate using keyed hash functions (e.g., HMAC) as PRFs. HMAC is a PRF if the underlying compression function of the hash function in use is a PRF [30]. When HMAC is used, $f_{k}(r)$ is the HMAC of $r$ with $k$ as the key.

The purpose of $h$ is to shorten a long bit string. It maps the output of $f_{k}$ to a shorter bit string of length $\alpha$, where $\alpha$ is the modulus size of $M$ (i.e., $\alpha=|M|$ ). $h$ is not required to be collision-consistent, but its output should be uniformly distributed over $\{0,1\}^{\alpha}$. An example construction for $h$ is to truncate the output of $f_{k}$ into shorter bit strings of length $\alpha$, take exclusive-OR on all these strings and use it as the output of $h$. This scheme is proved to be semantically secure [20].

This scheme allows additive homomorphic encryption. Given two ciphertexts $c_{1}=\left(m_{1}+h\left(f_{k}(r)\right)\right) \bmod M$ and $c_{2}=\left(m_{2}+h\left(f_{k^{\prime}}(r)\right)\right) \bmod M$, an individual that knows $k$ and $k^{\prime}$ can compute the sum of $m_{1}$ and $m_{2}$ directly from the aggregate ciphertext $c=c_{1}+c_{2}$ :

$$
m=m_{1}+m_{2}=\left(c-h\left(f_{k}(r)\right)-h\left(f_{k^{\prime}}(r)\right)\right) \bmod M .
$$

To correctly compute the sum of $n$ messages $m_{1}, m_{2}, \ldots, m_{n}$, $M$ must be larger than $\sum_{i=1}^{n} m_{i}$. In practice, $M$ should be selected as $M=2^{\left\lceil\log _{2}\left(\max \left(m_{i}\right) \cdot n\right)\right\rceil}$.

Table I shows the notations used in this paper.

\section{Aggregation Protocol for Sum}

\section{A. Protocol Overview}

Setup: The key dealer assigns a set of secret values (secrets for short) to each user and the aggregator.

Enc: In each time period, user $i(i \in[1, n])$ generates encryption key $k_{i}$ using the secrets that it is assigned. It encrypts its data $x_{i}$ by computing

$$
c_{i}=\left(k_{i}+x_{i}\right) \bmod M
$$


TABLE I

NOTATIONS

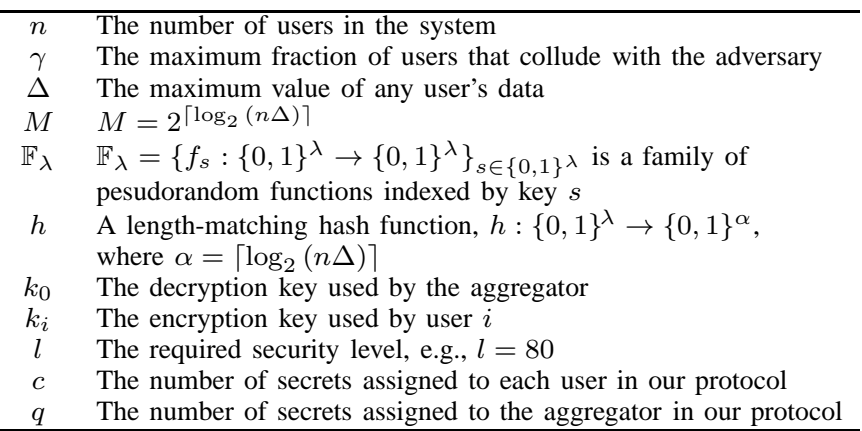

where $M=2^{\left\lceil\log _{2}(n \Delta)\right\rceil}$. Then it sends the ciphertext $c_{i}$ to the aggregator.

AggrDec: In each time period, the aggregator generates decryption key $k_{0}$ using the secrets that it is assigned, and decrypts the sum aggregate $S=\sum_{i=1}^{n} x_{i}$ by computing

$$
S=\left(\sum_{i=1}^{n} c_{i}-k_{0}\right) \quad \bmod M .
$$

The keys are generated using a PRF family and a lengthmatching hash function (see later). According to [29], the aggregator can get the correct sum so long as the following equation holds:

$$
k_{0}=\left(\sum_{i=1}^{n} k_{i}\right) \bmod M .
$$

In our protocol, the setup phase only runs once. After the setup phase, the key dealer does not need to distribute secrets to the users and the aggregator again. In addition, the users and the aggregator do not have to synchronize their key generations with communications in every time period. These restrictions make it challenging for the users and the aggregator to generate their keys such that Equation 3 holds in every time period and the encryption (decryption) key used by each user (the aggregator) cannot be learned by any other party besides the key dealer.

We propose a construction for key generations which preserves the privacy of each user and the Sum aggregate efficiently. Before presenting our construction, we first discuss a straw-man construction which is very efficient for the users but not efficient for the aggregator. Then we extend this strawman scheme to derive our construction.

Both constructions include three processes, which are secret setup, encryption key generation and decryption key generation. They proceed in the Setup phase, Enc phase and AggrDec phase of the aggregation protocol, respectively.

\section{B. A Straw-man Construction for Key Generation}

1) Intuition: Figure 2 shows the intuition of the strawman construction. Suppose there are $n c$ random numbers. The aggregator has access to all the numbers, and it computes the sum of these numbers as the decryption key $k_{0}$. These numbers are divided into $n$ random disjoint subsets, each of size $c$.

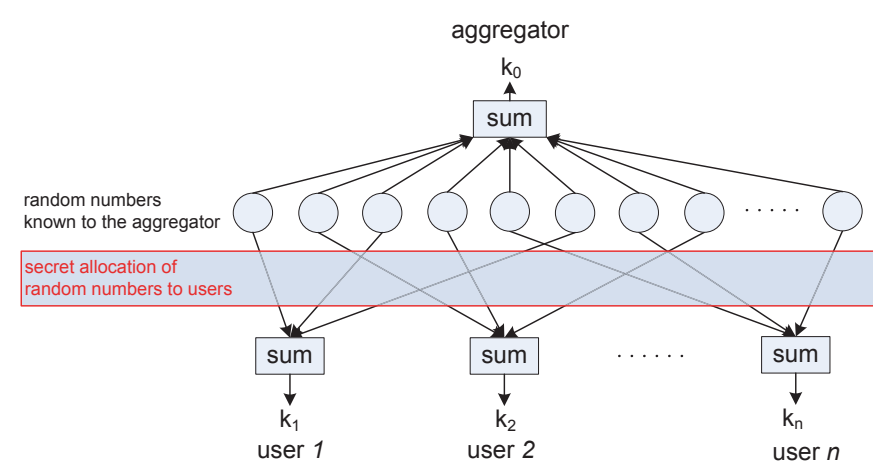

Fig. 2. The intuition behind the straw-man construction. The aggregator computes the sum of a set of random numbers as the decryption key. These numbers are secretly allocated to the users, and each user computes the sum of its allocated numbers as the encryption key. The aggregator does not know which random numbers are allocated to each user, and thus does not know any user's key.

These $n$ subsets are assigned to the $n$ users, where each user has access to one subset of numbers. User $i$ computes the sum of the numbers assigned to it as the encryption key $k_{i}$.

Clearly, Equation 3 holds. The aggregator cannot know any user's encryption key since it does not know the mapping between the random numbers and the users. When $c$ is large enough, it is infeasible for the aggregator to guess the numbers assigned to a particular user with a brute-force method. The aggregator's decryption key cannot be revealed by any user since no user knows all the numbers.

2) Construction: The construction is as follows:

Secret Setup: The key dealer generates $n c$ random and different secrets $s_{1}, \ldots, s_{n c}$. It divides these secrets into $n$ random disjoint subsets, with $c$ secrets in each subset. Let $\mathcal{S}$ denote the set of all secrets, and let $\mathcal{S}_{i}$ denote the $i^{\text {th }}$ subset. Clearly, $\mathcal{S}=\bigcup_{i=1}^{n} \mathcal{S}_{i}$ and $\forall i \neq j, \mathcal{S}_{i} \cap \mathcal{S}_{j}=\phi$. The key dealer sends the secrets in subset $\mathcal{S}_{i}$ to user $i$ and sends all the secrets in $\mathcal{S}$ to the aggregator.

Encryption Key Generation: In time period $t \in \mathbb{N}$, user $i$ generates its encryption key as follows:

$$
k_{i}=\left(\sum_{s^{\prime} \in \mathcal{S}_{i}} h\left(f_{s^{\prime}}(t)\right)\right) \bmod M .
$$

Decryption Key Generation: In time period $t \in \mathbb{N}$, the aggregator generates the decryption key as follows:

$$
k_{0}=\left(\sum_{s^{\prime} \in \mathcal{S}} h\left(f_{s^{\prime}}(t)\right)\right) \bmod M .
$$

In Equation 4, since each $h\left(f_{s^{\prime}}(t)\right)$ is uniformly distributed over $\{0,1\}^{\alpha}, k_{i}$ is also uniformly distributed over $\{0,1\}^{\alpha}$. Thus, the encryption keys satisfy the security requirement of the underlying cryptosystem. Equation 3 also holds since

$$
\begin{aligned}
\sum_{i=1}^{n} k_{i} & =\left(\sum_{i=1}^{n} \sum_{s^{\prime} \in \mathcal{S}_{i}} h\left(f_{s^{\prime}}(t)\right)\right) \bmod M \\
& =\left(\sum_{s^{\prime} \in \mathcal{S}} h\left(f_{s^{\prime}}(t)\right)\right) \quad \bmod M \\
& =k_{0} \bmod M .
\end{aligned}
$$


TABLE II

SECURITY LEVELS OF THE STRAW-MAN CONSTRUCTION WHEN $\gamma=0.1$.

\begin{tabular}{|c|cccccc|}
\hline$n=10^{2}$ & $c$ & 10 & 11 & 12 & 13 & 14 \\
& $p_{b}$ & $2^{-76.3}$ & $2^{-84.1}$ & $2^{-92.0}$ & $2^{-99.9}$ & $2^{-107.7}$ \\
\hline \hline$n=10^{3}$ & $c$ & 6 & 7 & 8 & 9 & 10 \\
& $p_{b}$ & $2^{-64.9}$ & $2^{-76.0}$ & $2^{-87.2}$ & $2^{-98.4}$ & $2^{-109.6}$ \\
\hline \hline$n=10^{4}$ & $c$ & 4 & 5 & 6 & 7 & 8 \\
& $p_{b}$ & $2^{-56.0}$ & $2^{-70.4}$ & $2^{-84.8}$ & $2^{-99.3}$ & $2^{-113.8}$ \\
\hline \hline$n=10^{5}$ & $c$ & 3 & 4 & 5 & 6 & 7 \\
& $p_{b}$ & $2^{-51.5}$ & $2^{-69.2}$ & $2^{-87.0}$ & $2^{-104.8}$ & $2^{-122.6}$ \\
\hline \hline$n=10^{6}$ & $c$ & 2 & 3 & 4 & 5 & 6 \\
& $p_{b}$ & $2^{-40.6}$ & $2^{-61.5}$ & $2^{-82.5}$ & $2^{-103.6}$ & $2^{-124.7}$ \\
\hline
\end{tabular}

TABLE III

THE MINIMUM VALUES OF $c$ FOR 80 -BIT SECURITY IN THE STRAW-MAN CONSTRUCTION.

\begin{tabular}{|c|ccccc|}
\hline$n$ & $10^{2}$ & $10^{3}$ & $10^{4}$ & $10^{5}$ & $10^{6}$ \\
\hline$\gamma=0,0.1,0.2,0.3$ & 11 & 8 & 6 & 5 & 4 \\
\hline
\end{tabular}

3) Security level: If the aggregator knows the $c$ secrets used by a user, it can obtain the encryption key of the user. We can derive the probability that the aggregator finds the $c$ secrets used by a user. Let $p_{b}$ denote the probability that in a single trial the aggregator can successfully guess the secrets assigned to the user. Recall that $\gamma$ is the maximal fraction of users that collude with the aggregator. In the worst case, the aggregator knows the $\gamma n c$ secrets assigned to the colluding users, but it does not know how the remaining $(1-\gamma) n c$ secrets are assigned to other users. There are $\left(\begin{array}{c}(1-\gamma) n c \\ c\end{array}\right)$ possible secret assignments for each user. Hence we have:

$$
p_{b}=\frac{1}{\left(\begin{array}{c}
(-\gamma) n c \\
c
\end{array}\right)} \text {. }
$$

With a smaller $p_{b}$, better security can be achieved. Table II shows the values of $p_{b}$ for varying parameters $n$ and $c$. As $c$ increases, the security level increases quickly.

Given the number of users $n$ and an estimate of $\gamma$, we can derive the minimum value of $c$ to achieve a certain required security level. ( $c$ is minimized to minimize the cost.) For $l$-bit security (e.g., $l=80$ ), $c$ should be selected as the minimum value that satisfies $p_{b} \leq 2^{-l}$. Table III shows the values of $c$ for 80-bit security.

A fraction of users may collude against the aggregator to reveal the aggregate. To achieve this goal, they need to obtain all the secrets that the aggregator has. However, each user only knows a subset of the secrets. So long as not all users collude, they cannot obtain all the secrets.

4) Cost: In each time period, each user computes $c$ PRFs and the aggregator computes $n c$ PRFs. Since $c$ is small as shown in Table III, the computation cost at each user is very low. However, when the number of users $n$ is very large, the computation cost at the aggregator is high.

\section{Our Construction for Key Generation}

Our construction extends the straw-man construction to reduce the computation overhead at the aggregator.

1) Intuition: Consider an equation:

$$
a_{1}+a_{2}+\cdots+a_{n c}=a_{1}+a_{2}+\cdots+a_{n c} .
$$

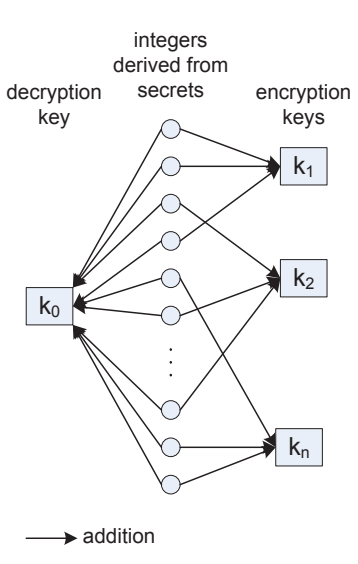

(a) Straw-man construction

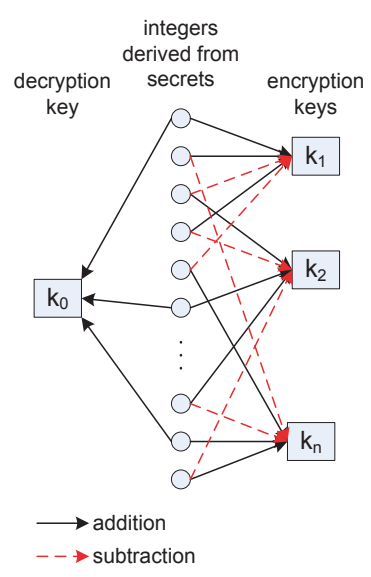

(b) Our construction
Fig. 3. The intuition behind our construction in comparison with the strawman construction.

If we remove $n c-q$ summands from the right side and subtract them from the left side, the derived equation

$a_{1}+\cdots+a_{n c}+\left(-a_{1}\right)+\cdots+\left(-a_{n c-q}\right)=a_{n c-q+1}+\cdots+a_{n c}$

is equivalent to the original equation.

To meet the requirement of Equation 3, the straw-man construction essentially mimics Equation 7, i.e., the users collectively generate the summands on the left side and add them to the aggregate, while the aggregator alone generates the summands on the right side and subtracts them from the perturbed aggregate (see Figure 3(a)). Each summand is generated from a secret. Since Equation 7 and 8 are equivalent, we can remove some summands from the aggregator side and subtract them from the user side without violating Equation 3 . Now the aggregator has less computation overhead since it needs to generate less summands. The reduced computation does not come for free, as it is amortized among the users such that each user generates more summands (see Figure 3(b)). A nice property is that it is now more difficult to guess the summands generated by each user and thus each user has better security.

2) Construction: The construction is as follows:

Secret Distribution: The key dealer generates $n c$ random and different secrets $s_{1}, \ldots, s_{n c}$. Let $\mathcal{S}$ denote the set composed of all the secrets. The key dealer divides these secrets into $n$ random disjoint subsets, with $c$ secrets in each subset. For convenience, we call these subsets additive subsets. Let $\mathcal{S}_{i}$ denote the $i^{\text {th }}$ additive subset. Clearly, $\mathcal{S}=\bigcup_{i=1}^{n} \mathcal{S}_{i}$.

Out of the $n c$ secrets, the key dealer randomly selects $q$ secrets and assigns them to the aggregator. Let $\hat{\mathcal{S}}$ denote the set of secrets assigned to the aggregator. The key dealer divides the remaining $n c-q$ secrets evenly into $n$ random disjoint subsets. Among them, $(n c-q)-n\left\lfloor\frac{n c-q}{n}\right\rfloor$ subsets have $\left\lfloor\frac{n c-q}{n}\right\rfloor+1$ secrets each, and the other $n\left(1+\left\lfloor\frac{n c-q}{n}\right\rfloor\right)-n c+q$ subsets have $\left\lfloor\frac{n c-q}{n}\right\rfloor$ secrets each. For convenience, we call these subsets subtractive subsets. Let $\overline{\mathcal{S}}_{i}$ denote the $i^{t h}$ subtractive subset. Clearly, $\mathcal{S}=\left(\bigcup_{i=1}^{n} \overline{\mathcal{S}}_{i}\right) \bigcup \hat{\mathcal{S}}$. The key dealer assigns the secrets in the additive subset $\mathcal{S}_{i}$ and subtractive subset $\overline{\mathcal{S}}_{i}$ to user $i$. 
TABLE IV

THE SECURITY LEVEL OF OUR CONSTRUCTION WHEN $\gamma=0.1$.

\begin{tabular}{|c|cccccc|}
\hline$n=10^{2}$ & $c$ & 4 & 5 & 6 & 7 & 8 \\
& $p_{b}$ & $2^{-51.0}$ & $2^{-66.5}$ & $2^{-82.1}$ & $2^{-97.7}$ & $2^{-113.3}$ \\
\hline \hline$n=10^{3}$ & $c$ & 3 & 4 & 5 & 6 & 7 \\
& $p_{b}$ & $2^{-52.2}$ & $2^{-74.3}$ & $2^{-96.4}$ & $2^{-118.7}$ & $2^{-140.9}$ \\
\hline \hline$n=10^{4}$ & $c$ & 2 & 3 & 4 & 5 & 6 \\
& $p_{b}$ & $2^{-40.4}$ & $2^{-68.8}$ & $2^{-97.5}$ & $2^{-126.3}$ & $2^{-155.2}$ \\
\hline \hline$n=10^{5}$ & $c$ & 1 & 2 & 3 & 4 & 5 \\
& $p_{b}$ & $2^{-16.5}$ & $2^{-50.4}$ & $2^{-85.5}$ & $2^{-120.8}$ & $2^{-156.2}$ \\
\hline \hline$n=10^{6}$ & $c$ & 1 & 2 & 3 & 4 & 5 \\
& $p_{b}$ & $2^{-19.8}$ & $2^{-60.3}$ & $2^{-102.1}$ & $2^{-144.0}$ & $2^{-186.1}$ \\
\hline
\end{tabular}

Encryption Key Generation: In time period $t \in \mathbb{N}$, user $i$ generates its encryption key by computing

$$
k_{i}=\left(\sum_{s^{\prime} \in \mathcal{S}_{i}} h\left(f_{s^{\prime}}(t)\right)-\sum_{s^{\prime} \in \overline{\mathcal{S}}_{i}} h\left(f_{s^{\prime}}(t)\right)\right) \quad \bmod M .
$$

Decryption Key Generation: In time period $t \in \mathbb{N}$, the aggregator generates the decryption key by computing

$$
k_{0}=\left(\sum_{s^{\prime} \in \hat{\mathcal{S}}} h\left(f_{s^{\prime}}(t)\right)\right) \quad \bmod M .
$$

The requirement in Equation 3 is satisfied since

$$
\begin{aligned}
\sum_{i=1}^{n} k_{i} & =\left(\sum_{i=1}^{n}\left(\sum_{s^{\prime} \in \mathcal{S}_{i}} h\left(f_{s^{\prime}}(t)\right)-\sum_{s^{\prime} \in \overline{\mathcal{S}}_{i}} h\left(f_{s^{\prime}}(t)\right)\right)\right) \bmod M \\
& =\left(\sum_{s^{\prime} \in \mathcal{S}} h\left(f_{s^{\prime}}(t)\right)-\sum_{s^{\prime} \in \bigcup_{i=1}^{n} \overline{\mathcal{S}}_{i}} h\left(f_{s^{\prime}}(t)\right)\right) \bmod M \\
& =\left(\sum_{s^{\prime} \in \hat{\mathcal{S}}} h\left(f_{s^{\prime}}(t)\right)\right) \bmod M \\
& =k_{0} .
\end{aligned}
$$

3) Security level: The aggregator cannot learn any user's encryption key since it does not know the additive secrets (i.e., secrets in the additive subset) and the subtractive secrets (i.e., secrets in the subtractive subset) assigned to this user. Each user has $c$ additive secrets and at least $\left\lfloor\frac{n c-q}{n}\right\rfloor$ subtractive secrets. The aggregator may know the secrets assigned to itself and those to its $\gamma n$ colluders, but there are still $(1-\gamma) n c$ additive secrets and at least $(1-\gamma) n\left\lfloor\frac{n c-q}{n}\right\rfloor$ subtractive secrets that the aggregator does not know how they are assigned to the good users. There are at least $\left(\begin{array}{c}(1-\gamma) n c \\ c\end{array}\right) \cdot\left(\begin{array}{c}(1-\gamma) n\left\lfloor\frac{n c-q}{n}\right\rfloor \\ \left\lfloor\frac{n c-q}{n}\right\rfloor\end{array}\right)$ possible secret assignments for each good user. Thus,

$$
p_{b} \leq \frac{1}{\left(\begin{array}{c}
1-\gamma) n c \\
c
\end{array}\right) \cdot\left(\begin{array}{c}
1-\gamma) n\left\lfloor\frac{n c-q}{n}\right\rfloor \\
\left\lfloor\frac{n c-q}{n}\right\rfloor
\end{array}\right)} .
$$

$p_{b}$ decreases (i.e., the security for the users is better) when $n$ and $c$ increase, but $p_{b}$ increases when $\gamma$ increases.

Under the same total computation cost, the smaller $q$ is, the more subtractive secrets the users are assigned and the better security the users have. However, if $q$ is too small, the secrets (and hence the decryption key) used by the aggregator may be learned by a number of colluding users in the brute-force way. We can derive the minimum value of $q$ to make it infeasible
TABLE $\mathrm{V}$

THE VALUES OF $c$ FOR 80 -BIT SECURITY IN OUR CONSTRUCTION.

\begin{tabular}{|c|ccccc|}
\hline$n$ & $10^{2}$ & $10^{3}$ & $10^{4}$ & $10^{5}$ & $10^{6}$ \\
\hline \hline$\gamma=0,0.1,0.2$ & 6 & 5 & 4 & 3 & 3 \\
\hline$\gamma=0.3$ & 7 & 5 & 4 & 3 & 3 \\
\hline
\end{tabular}

TABLE VI

THE VALUES OF $q$ FOR 80 -BIT SECURITY IN OUR CONSTRUCTION.

\begin{tabular}{|c|ccccc|}
\hline$n$ & $10^{2}$ & $10^{3}$ & $10^{4}$ & $10^{5}$ & $10^{6}$ \\
\hline \hline$\gamma=0$ & 12 & 8 & 6 & 5 & 4 \\
\hline$\gamma=0.1$ & 13 & 8 & 6 & 5 & 4 \\
\hline$\gamma=0.2$ & 13 & 8 & 6 & 5 & 4 \\
\hline$\gamma=0.3$ & 13 & 9 & 7 & 5 & 5 \\
\hline
\end{tabular}

for $\gamma$ fraction of users to collusively obtain the decryption key. These colluders know at most $\gamma n c$ subtractive secrets, but they do not know which $q$ of the remaining $(1-\gamma) n c$ secrets the aggregator has. There are $\left(\begin{array}{c}(1-\gamma) n c \\ q\end{array}\right)$ possible secret assignments for the aggregator. Let $p_{c}$ denote the probability that the $q$ secrets assigned to the aggregator can be guessed in a single trial. We have

$$
p_{c} \leq \frac{1}{\left(\begin{array}{c}
(1-\gamma) n c \\
q
\end{array}\right)} .
$$

When $q$ increases, $p_{c}$ decreases which means better security for the aggregator.

4) Practical considerations: To achieve l-bit security for each user and the aggregator, it is required that $p_{b} \leq 2^{-l}$ and $p_{c} \leq 2^{-l}$ respectively. Given parameters $n$ and $\gamma$, large-enough values should be set for $c$ and $q$ to meet these requirements.

Since the values of $c$ and $q$ depend on each other, which can be seen from Equation 11 and 12, they can be set as follows. First, we assume $q \in(0, n]$. Under this assumption, Equation 11 can be rewritten as:

$$
p_{b} \leq \frac{1}{\left(\begin{array}{c}
(1-\gamma) n c \\
c
\end{array}\right) \cdot\left(\begin{array}{c}
(1-\gamma) n(c-1) \\
c-1
\end{array}\right)} .
$$

We can derive the minimum value of $c$ that makes the righthand side of Equation 13 smaller than $2^{-l}$. Then we apply the derived value of $c$ to Equation 12, and obtain the minimum value of $q$ that makes the right-hand side of Equation 12 smaller than $2^{-l}$. If the obtained value of $q$ falls into the assumed range $(0, n]$, the values of $c$ and $q$ are accepted. Otherwise, we can increase the value of $c$, until the minimum value of $q$ that makes the right-hand side of Equation 12 smaller than $2^{-l}$ is not larger than $n$.

This method of setting $c$ and $q$ ensures that $q \leq n$, and thus $p_{b}$ is given in Equation 13. Table IV shows the values of $p_{b}$ when $n$ and $c$ change. Table $\mathrm{V}$ and Table VI show the values of $c$ and $q$ respectively for 80-bit security. It can be seen that both $c$ and $q$ are very small.

5) Cost: Since the setup phase is run only once, we analyze the cost of our construction in each aggregation period. The computation cost is measured by the number of PRFs computed, since the length-matching hash function (which mainly consists of exclusive-OR operations) and arithmetic addition are much less expensive in computation.

In each time period, each user computes $2 c-\frac{q}{n}$ PRFs on 
TABLE VII

THE SECURITY AND COST OF OUR CONSTRUCTION AND THE STRAW-MAN CONSTRUCTION. FOR COMPUTATION COST, THE VALUE IS THE COST PER TIME PERIOD.

\begin{tabular}{|c|c|c|}
\hline & Straw-man & Ours \\
\hline$p_{b}$ & $\frac{1}{\left(\begin{array}{c}1-\gamma) n c \\
c\end{array}\right.}$ & $\frac{1}{\left(\begin{array}{c}(1-\gamma) n c \\
c\end{array}\right) \cdot\left(\begin{array}{c}(1-\gamma) n(c-1) \\
c-1\end{array}\right)}$ \\
\hline Comp. (total) & $2 n c$ & $2 n c$ \\
\hline Comp. (user) & $c$ & $2 c-\frac{q}{n}$ \\
\hline $\begin{array}{c}\text { Comp. } \\
\text { (aggregator) }\end{array}$ & $n c$ & $q(q<n)$ \\
\hline Storage (user) & $c$ & $2 c-q$ \\
\hline $\begin{array}{c}\text { Storage } \\
\text { (aggregator) }\end{array}$ & $n c$ & $q$ \\
\hline
\end{tabular}

TABLE VIII

THE COMPUTATION COST OF OUR CONSTRUCTION AND THE STRAW-MAN CONSTRUCTION FOR 80-BIT SECURITY WHEN $\gamma=0.1$.

\begin{tabular}{|c|c|ccccc|}
\hline & $n$ & $10^{2}$ & $10^{3}$ & $10^{4}$ & $10^{5}$ & $10^{6}$ \\
\hline \hline User & Straw-man & 11 & 8 & 6 & 5 & 4 \\
& Ours & 12 & 10 & 8 & 6 & 6 \\
\hline Aggregator & Straw-man & 1100 & 8000 & $6 \cdot 10^{4}$ & $5 \cdot 10^{5}$ & $4 \cdot 10^{6}$ \\
& Ours & 13 & 8 & 6 & 5 & 4 \\
\hline
\end{tabular}

average, while the aggregator computes $q$ PRFs. As for the storage cost, the key dealer stores $n c$ secrets as well as $2 n c$ mappings between the secrets and the users/aggregator. Each user stores $2 c-\frac{q}{n}$ secrets on average, while the aggregator stores $q$ secrets. Besides sending the encrypted data to the aggregator, each user does not make any extra communications.

6) Comparisons with the Straw-man Construction: Table VII compares our construction to the straw-man construction in security and cost. When the total computation cost (for users and the aggregator) is the same, our construction achieves better security. Also, it has smaller computation cost at the aggregator. Upon initial inspection, our construction may seem to double the computation cost at each user (i.e., from $c$ to roughly $2 c$ ). In practice, however, it can use a smaller $c$ to achieve the same security level. Table VIII shows the computation cost of the two constructions at the same security level. For a wide range of $n\left(10^{2}-10^{6}\right)$, the computation cost at each user is slightly higher (i.e., one or two PRFs) in our construction, but the computation cost at the aggregator is orders of magnitude smaller.

\section{Aggregation Protocol for Min}

The Min aggregate is defined as the minimum value of the users' data. This section presents a protocol which employs the Sum aggregate to get Min.

\section{A. The Basic Scheme}

This scheme gets the Min aggregate of each time period using $\Delta+1$ parallel Sum aggregates in the same time period. The sums used to obtain Min are based on a number of 1-bit derivative data (denoted by $d$ ) derived from the users' raw data $x$. Without loss of generality, we assume $\Delta$ is a power of two.

The scheme works as follows. In each time period, each user generates $\Delta+1$ derivative data $d[0], d[1], \ldots, d[\Delta]$, where each derivative data corresponds to one possible data value in the

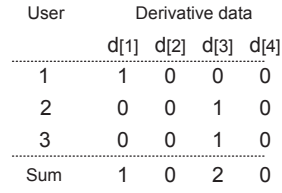

(a) Original derivative data (b) Extended and concatenated data

\begin{tabular}{|c|c|c|c|c|c|c|c|c|c|c|}
\hline \multirow[t]{2}{*}{ User } & \multicolumn{10}{|c|}{ Derivative data } \\
\hline & 1 & [1] & । & $\mathrm{d}[2]$ & & & & । & $\mathrm{d}[4$ & \\
\hline 1 & 10 & 1 & $!$ & $c$ & & 0 & 0 & 1 & 0 & 0 \\
\hline 2 & 0 & 0 & ( & c & & 0 & 1 & i & 0 & 0 \\
\hline 3 & 10 & 0 & : & 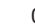 & & 0 & 1 & & 0 & 0 \\
\hline Sum & 0 & 1 & i & ( & & 1 & 0 & & 0 & 0 \\
\hline
\end{tabular}

Fig. 4. An example of sum based on derivative data.

plaintext space. For each $j \in[0, \Delta]$, the user assigns 1 to $d[j]$ if its raw data value is equal to $j$ and assigns 0 otherwise. For each $j \in[0, \Delta]$, the aggregator can obtain the Sum aggregate of $d[j]$ using the sum aggregation protocol presented in Section IV. Then Min is the smallest $j$ that returns a positive sum.

In each time period, each user involves in $\Delta+1$ sum aggregates over $\Delta+1$ derivative data. Note that in the sum aggregation protocol each user computes $2 c$ PRFs to encrypt her data. It is inefficient to compute $2 c$ PRFs for each derivative data. Since these data are independent, we use a more efficient technique that concatenates multiple data together and encrypts them as a whole.

This technique extends each derivative data from one bit to $\lceil\log (n+1)\rceil$ bits by adding $\lceil\log (n+1)\rceil-10$ 's on the left, and then concatenates all extended derivative data into a single bit string. The sum of the concatenated string (interpreted as an integer) is obtained using the sum aggregation protocol. The obtained sum is considered as a bit string, and split into substrings of $\lceil\log (n+1)\rceil$ bits each. Each substring, when interpreted as an integer, represents the sum of one derivative data. Note that these substrings do not affect each other (i.e., no carries among them), since the sum of each derivative data does not exceed $n$. Figure 4 shows an example of this process.

Clearly, the concatenated data has $(\Delta+1)\lceil\log (n+1)\rceil$ bits. The ciphertext generated by each user has $\alpha=(\Delta+$ 1) $\lceil\log (n+1)\rceil$ bits. If $\alpha$ is larger than $H$, which is the size of the output generated by the PRF (i.e., an HMAC), we can divide the derivative data into $\frac{(\Delta+1)\lceil\log (n+1)\rceil}{H}$ groups and apply the above technique to each group in parallel. Thus, $\frac{(\Delta+1)\lceil\log (n+1)\rceil}{H}$ parallel instances of the sum aggregation protocol are needed in each time period. For example, when $n=1000, \Delta=10000$ and SHA-512 is used as the hash function of HMAC, 196 instances of the sum aggregation protocol are needed.

Each user uses just one set of secrets for all instances of the sum aggregation protocol. For instance $j$, it uses $h\left(f_{s^{\prime}}(j \mid t)\right)$ to generate the encryption key instead of using $h\left(f_{s^{\prime}}(t)\right)$ in the original protocol (see Equation 9). Similarly, the aggregator also uses just one set of secrets.

Since the sum aggregation protocol does not expose the derivative data of any user, the aggregator cannot know the data value of any specific user.

\section{B. Low-cost Min Aggregation}

When the plaintext space is large, the cost of the basic scheme is high. In some application scenarios, it may not be necessary to get the exact Min, but an approximate answer 
is good enough. For such scenarios, the basic scheme can be extended to get an approximate Min with much smaller cost.

Specifically, we wish to obtain an approximate Min where the relative error (defined as $\frac{\mid \text { Exact Min-Approximate Min } \mid}{\max \{\text { Exact Min, } 1\}}$ ) is required to be lower than $\frac{1}{2^{\epsilon}}(\epsilon \geq 0)$. To meet this requirement, the exact value of Min should be obtained if Min is smaller than or equal to $2^{\epsilon}$, and the $\epsilon$-bit segment of Min (when Min is interpreted as a bit string) that starts from the first ' 1 ' bit should be obtained if Min is larger than $2^{\epsilon}$. For example, suppose Min is 42 (00101010) out of 8-bit data. To make the relative error smaller than $\frac{1}{2^{3}}$, it is sufficient to know that Min has the bit pattern 00101xxx. Then we can set the bit that follows the known bits as 1 and set other bits as 0 . The obtained approximate Min is 00101100, which is 44 . The relative error is $\frac{1}{21}$, which is smaller than the required $\frac{1}{2^{3}}$.

To obtain the approximate Min, each user appends $\epsilon+1$ padding bits to its raw data. If the data value is zero, the first padding bit is 1 and the others are 0 ; otherwise, all the padding bits are 0 . The padded data has $\log \Delta+\epsilon+2$ bits and at least one bit is 1 . The first ' 1 ' bit of Min may appear in any of the first $\log \Delta+2$ bits of the padded data. In the case it appears at the first padding bit, Min is zero.

Suppose in the binary representation of data value, the weight of bit decreases from the left to the right. Let $\delta$ $(\delta \in[1, \log \Delta+2])$ denote the location (indexed from the left) of the first ' 1 ' bit of Min. Smaller $\delta$ means larger Min. Let $\sigma$ denote the value of the $(\epsilon-1)$-bit segment of Min that follows $\delta$. When $\delta$ is the same, a larger $\sigma$ means larger Min. Clearly, there are $2^{\epsilon-1}(\log \Delta+2)$ possible combinations of $\langle\delta, \sigma\rangle$. We map these combinations to an auxiliary plaintext space $0,1, \ldots, 2^{\epsilon-1}(\log \Delta+2)-1$, such that if one combination means smaller Min than another combination, it is mapped to a smaller value in the auxiliary plaintext space than that combination. Let $v[\delta, \sigma]$ denote the value that combination $\langle\delta, \sigma\rangle$ maps to.

In each time period, each user converts its padded raw data to a value $x^{\prime}$ in the auxiliary plaintext space as follows: if in its padded raw data the first ' 1 ' bit appears at $\delta^{\prime}$ and the value of the $(\epsilon-1)$-bit segment that follows the first ' 1 ' bit is $\sigma^{\prime}$, it sets $x^{\prime}=v\left[\delta^{\prime}, \sigma^{\prime}\right]$. The aggregator can get the Min of $x^{\prime}$ using the basic scheme, and reversely map the Min of $x^{\prime}$ to a pair of $\langle\delta, \sigma\rangle$. It knows that the first ' 1 ' bit of the Min aggregate over padded raw data appears at location $\delta$, and the $(\epsilon-1)$-bit segment that follows the first ' 1 ' bit is $\sigma$. Then it sets the bit that follows the $(\epsilon-1)$-bit segment as 1 , and sets the remaining bits as 0 . This derives the Min aggregate over padded raw data, which has the form $\{0\}^{\delta-1}\{1\}^{1}\{0,1\}^{\epsilon-1}\{1\}^{1}\{0\}^{\log \Delta+2-\delta}$. From this bit string, the last $\epsilon+1$ padding bits are removed and then the approximate Min of users' raw data is obtained. Figure 5 shows a running example of this process.

In total, this scheme uses $2^{\epsilon-1}(\log \Delta+2)$ parallel Sum aggregates of 1-bit derivative data. Thus, in each time period, $\frac{2^{\epsilon-1}(\log \Delta+2)}{H}$ parallel instances of the sum aggregation protocol are needed, and each user sends $2^{\epsilon-1}(\log \Delta+2)$ bits of encrypted data (which can be encapsulated into one message)
TABLE IX

THE RELATIVE ERROR AND COST OF THE BASIC SCHEME AND THE LOW-COST SCHEME.

\begin{tabular}{|c|c|c|}
\hline & Basic & Low-cost \\
\hline Relative error & 0 & $\leq \frac{1}{2^{\epsilon}}$ \\
Encryption (PRFs) & $\frac{2 c(\Delta+1)\lceil\log (n+1)\rceil}{H}$ & $\frac{2^{\epsilon} c(\log \Delta+2)}{H}$ \\
Decryption (PRFs) & $\frac{q(\Delta+1)\lceil\log (n+1)\rceil}{H}$ & $\frac{2^{\epsilon-1} q(\log \Delta+2)}{H}$ \\
Ciphertext size (bit) & $(\Delta+1)\lceil\log (n+1)\rceil$ & $2^{\epsilon-1}(\log \Delta+2)$ \\
\hline
\end{tabular}

$H$ is the size of the output generated by the PRF.

to the aggregator. For example, when $\Delta=10^{4}$, SHA-512 is used as the hash function of HMAC and it is required to limit the relative error to $1 \%$ (i.e., $\epsilon=7$ ), only 2 instances are needed and each user only sends 128 bytes of ciphertexts to the aggregator in each time period.

Table IX summarizes the relative error and cost of the basic scheme and the low-cost scheme.

\section{Dealing with Dynamic Joins and Leaves}

In mobile sensing applications, users may join and leave. When a user joins, it should be assigned some secrets for encryption key generation. When a user leaves, its secrets should be reclaimed such that the aggregator can still get the aggregate statistics of the remaining users. Dynamic joins and leaves should be properly dealt with to protect each user's privacy and ensure the secrecy of the aggregate statistics.

When the number of users is not large and the churn rate is low, the key dealer can re-run the secret setup phase for all the users whenever a user joins or leaves. However, for the applications with a large number of users and/or a high churn rate, the communication overhead may be too high to redistribute secrets to all users. In this section, we propose efficient techniques to deal with dynamic joins and leaves for a large-scale system. Basically, we use redundancy in security to reduce the communication overhead of joins and leaves.

For simplicity, we evaluate the communication overhead of dealing with a user's join and leave by the number of users that the key dealer should redistribute secrets to (or the number of updated users for short). Since the number of secrets redistributed to each user is not large, if we assume that these secrets can be included in one message, the number of updated users is equivalent to the number of messages that should be transmitted from the key dealer to the users.

For simplicity, we only consider the Sum protocol when describing our scheme to deal with dynamic joins and leaves, but the scheme applies to the protocol for Min as well.

\section{A. Challenges and Basic Idea}

Note that we consider a strong adversary who can monitor the communications between all entities including the key dealer and users. Through eavesdropping the message sending and receiving activities, the adversary can know which user joins or leaves and to which users secrets are redistributed to.

We use leave as an example to show the challenges of addressing user dynamics. When a user leaves, the key dealer needs to redistribute the user's secrets to other users and/or the aggregator, such that the aggregator can correctly decrypt the aggregate statistic of the remaining users in the future. Some 


\begin{tabular}{|c|c|c|c|c|c|c|c|}
\hline & $x[1]$ & $x[2]$ & $x[3] !$ & $x[4]$ & $x[5]$ & $x[6]$ & $x[7]$ \\
\hline$x_{1}=4$ & 1 & 0 & 0 & 0 & 0 & 0 & 0 \\
\hline$x_{2}=4$ & 1 & 0 & 0 & 0 & 0 & 0 & 0 \\
\hline$x_{3}=3$ & 0 & 1 & 1 & 0 & 0 & 0 & 0 \\
\hline$x_{4}=1$ & 0 & 0 & 1 & 0 & 0 & 0 & 0 \\
\hline
\end{tabular}

(a) Padded raw data

\begin{tabular}{|c|c|c|c|c|c|c|c|c|c|c|c|c|c|c|c|c|}
\hline$\delta$ & \multicolumn{4}{|c|}{1} & \multicolumn{4}{|c|}{2} & & \multicolumn{4}{|c|}{4} \\
\hline$\sigma$ & 11 & 10 & 01 & 00 & 11 & 10 & 01 & 00 & 11 & 10 & 01 & 100 & 11 & 10 & 01 & 00 \\
\hline$v[\delta, \sigma]$ & 15 & 14 & 13 & 12 & 11 & 10 & 9 & 8 & 7 & 6 & 5 & 4 & 3 & 2 & 1 & 0 \\
\hline$x_{1}=4$ & 0 & 0 & 0 & 1 & 0 & 0 & 0 & 0 & 0 & 0 & 0 & 0 & 0 & 0 & 0 & 0 \\
\hline$x_{2}=4$ & 0 & 0 & 0 & 1 & 0 & 0 & 0 & 0 & 0 & 0 & 0 & 0 & 0 & 0 & 0 & 0 \\
\hline$x_{3}=3$ & 0 & 0 & 0 & 0 & 0 & 1 & 0 & 0 & 0 & 0 & 0 & 0 & 0 & 0 & 0 & 0 \\
\hline$x_{4}=1$ & 0 & 0 & 0 & 0 & 0 & 0 & 0 & 0 & 0 & 0 & 0 & 1 & 0 & 0 & 0 & 0 \\
\hline Sum & 0 & 0 & 0 & 2 & 0 & 1 & 0 & 0 & 0 & 0 & 0 & 1 & 0 & 0 & 0 & 0 \\
\hline
\end{tabular}

(b) Derivative data

Fig. 5. An example of the process that obtains an approximate Min, where $\Delta=4$ and $\epsilon=3$. The four users' auxiliary data values are $x_{1}=12, x_{2}^{\prime}=12$, $x_{3}^{\prime}=10$ and $x_{4}^{\prime}=4$. The Min of the auxiliary data is 4 , and it is reversely mapped to $\langle\delta, \sigma\rangle=\langle 3,00\rangle$. Thus, the approximate Min of padded raw data is 0010010. After the last four padding bits are removed, the output is 001 .

of these secrets may be exposed to the adversary if they are redistributed to a user that colludes with the adversary. Then the adversary knows that these secrets belonged to the leaving user. As a result, there is less uncertainty about the secrets that the leaving user used before, and hence it is easier for the adversary to guess the user's past encryption key and infer her past data. Also, the adversary knows that these secrets do not belong to other users. This reduces the uncertainty about the secrets that the other users used before. Hence, it is easier to guess the encryption key of other users as well. Instead of only redistributing the leaving user's secrets, the key dealer may consider redistributing the secrets of the leaving user and a subset of other users, but the consequence will be similar. In both cases, the security level of past user data decreases. ${ }^{1}$ Thus, if each user maintains the minimum number of secrets needed for the required security level, which is case in the Sum aggregation protocol presented in Section IV, when a user leaves, the security of itself and other users will drop below the required level, unless a new set of secrets are distributed to all remaining users.

To address this problem, our idea is to let each user maintain higher than $l$-bit security by using more secrets than required by $l$-bit security. When a users joins and leaves, although some secrets may be exposed to the aggregator in the process of redistributing secrets, each user still maintains sufficient secrets for $l$-bit security, i.e., the required security level.

\section{B. Scheme Overview}

For convenience, we define the following three terms.

Definition 1: Affiliation The affiliation of a secret is the user that it is assigned to.

Definition 2: Forward security After the join (leave) operation, the key that an entity will use in the future is secure, i.e., the probability that the adversary can successfully guess the key in a single trial is not higher than $2^{-l}$.

Definition 3: Backward security After the join (leave) operation, the key that an entity used in the past is still secure, i.e., the probability that the adversary can successfully guess the key in a single trial is not higher than $2^{-l}$.

Let $\mathcal{D}$ denote the set of secrets which have been distributed to the users. These secrets are classified into two categories:

\footnotetext{
${ }^{1}$ Security can be maintained if all secrets are redistributed among all remaining users, but this is equivalent to rerunning the setup phase, a case that we want to avoid.
}

- Black secrets: A secret is black if its affiliation is not exposed to the adversary. That is, the adversary does not know the user that the secret belongs to. Let $\mathcal{B}$ denote the set of black secrets, $\mathcal{B}_{a}$ denote the set of black additive secrets and $\mathcal{B}_{s}$ denote the set of black subtractive secrets. (See the definitions of additive and subtractive secrets in Section IV-C2.) Clearly, $\mathcal{B}=\mathcal{B}_{a} \cup \mathcal{B}_{s}$.

- White secrets: A secret is white if its affiliation is exposed to the adversary. Let $\mathcal{W}$ denote the set of white secrets.

Obviously, $\mathcal{B} \cap \mathcal{W}=\phi$ and $\mathcal{B} \cup \mathcal{W}=\mathcal{D}$.

The security of a user depends on the number of black secrets it has and the total number of black secrets. Let $n_{a}\left(n_{s}\right)$ denote the number of black additive (subtractive) secrets that the user has. Then the probability that the adversary can guess the user's secrets in a single trial is given by $p_{b}=\frac{1}{\left(\begin{array}{c}\left|\mathcal{B}_{a}\right| \\ n_{a}\end{array}\right)\left(\begin{array}{l}\left|\mathcal{B}_{s}\right| \\ n_{s}\end{array}\right)}$. To maintain $l$-bit security, it is sufficient to ensure that $p_{b} \leq$ $2^{-l}$. That is,

$$
\left(\begin{array}{c}
\left|\mathcal{B}_{a}\right| \\
n_{a}
\end{array}\right)\left(\begin{array}{c}
\left|\mathcal{B}_{s}\right| \\
n_{s}
\end{array}\right) \geq 2^{l}
$$

Similarly, to maintain $l$-bit security for the aggregator, it is sufficient to ensure that

$$
\left(\begin{array}{c}
\left|\mathcal{B}_{a}\right| \\
q
\end{array}\right) \geq 2^{l}
$$

where $q$ is the number of black additive secrets that the aggregator has.

Due to user dynamics and the lack of knowledge on which users are colluding with the adversary, it is difficult to track the accurate values of $\left|\mathcal{B}_{a}\right|,\left|\mathcal{B}_{s}\right|, n_{a}$ and $n_{s}$. For simplicity, we address this problem by maintaining a lower bound for these parameters. Specifically, we ensure that

- Requirement 1: The total number of black additive (subtractive) secrets is at least $b$.

- Requirement 2: The number of black additive (subtractive) secrets that each user has is at least $x$.

Here, parameters $b$ and $x$ satisfy the following conditions:

$$
\begin{gathered}
\left(\begin{array}{l}
b \\
x
\end{array}\right)\left(\begin{array}{l}
b \\
x
\end{array}\right) \geq 2^{l} \\
\left(\begin{array}{l}
b \\
q
\end{array}\right) \geq 2^{l}
\end{gathered}
$$

Equation 16 guarantees the secrecy of each user's data and Equation 17 guarantees the secrecy of the aggregate statistic. 
TABLE $X$

NOTATIONS USED TO DEAL WITH DYNAMIC JOINS AND LEAVES

\begin{tabular}{cl}
\hline$b$ & The minimum total number of black additive (subtractive) secrets \\
$\varphi$ & The number of helper users used to deal with each join and leave \\
$x$ & The number of black additive (subtractive) secrets moved from each \\
& helper user to the joining user. It is also the minimum number of \\
black additive (subtractive) secrets that each user has \\
$x^{\prime}$ \\
The number of secrets removed from each helper user that are \\
redistributed along with the leaving user's secrets
\end{tabular}

In the following, we describe the detailed scheme to deal with dynamic joins and leaves which can meet Requirement 1 and Requirement 2. Besides the notations in Table I, other notations used in our scheme are summarized in Table X.

\section{The Detailed Scheme}

In this scheme, when a user joins, some secrets are moved from a random subset of existing users to the joining user. When a user leaves, the secrets of the leaving user are redistributed to a random subset of remaining users. In these operations, the random subset of existing and remaining users are called helper users for convenience.

1) Setup: The key dealer assigns secrets to each user and the aggregator as done in the construction presented in Section IV-C. Each user receives $c$ additive secrets and $c$ or $c-1$ subtractive secrets, and the aggregator receives $q$ additive secrets. Here, $c>x, n c-q>b$ and $\left(\begin{array}{l}b \\ q\end{array}\right) \geq 2^{l}$. All the secrets are tagged as black.

2) Join: When a user joins, the key dealer runs the following procedure.

- It randomly selects $\varphi$ helper users from the set of users that participated in the previous setup phase, where $\varphi$ is large enough to ensure that, given $\gamma$ (i.e., the maximum possible fraction of users colluding with the adversary), at least one helper user is good (i.e., not colluding with the adversary). If any helper user has less than $2 x$ black additive (subtractive) secrets, the key dealer runs the setup phase again.

- It removes $x$ black additive (subtractive) secrets from each helper user and assigns them to the new user.

- It tags the new user's secrets as white. Note that the new user will not be used as a helper user to deal with future joins and leaves.

- It checks that Requirement 1 is still satisfied. Note that there are at most $\gamma n$ users colluding with the adversary (where $n$ is the number of users in the system). The key dealer finds the $(1-\gamma) n$ users which have the minimum number of black additive secrets, and counts the number of additive secrets held by these users. If this number is smaller than $b$, it reruns the setup phase. Similarly, the key dealer can count the minimum possible number of black subtractive secrets. If this number is smaller than $b$, it also reruns the setup phase.

Forward security analysis. The last step ensures that Requirement 1 is satisfied after the join operation. Clearly, each helper user still has no less than $x$ black additive (subtractive) secrets after the join operation. Since at least one helper user is good, the new user also has at least $x$ black additive (subtractive) secrets. Requirement 2 is also satisfied. Thus, forward security is guaranteed for all users.

Backward security analysis. Since the total number of black additive (subtractive) secrets is larger before the join operation, Requirement 1 is satisfied before the join operation. Obviously, Requirement 2 is also satisfied. Hence, backward security is also guaranteed.

3) Leave: Let $\mathcal{S}_{1}\left(\mathcal{S}_{2}\right)$ denote the set of additive (subtractive) secrets the leaving user has. Let $n_{1}\left(n_{2}\right)$ denote the number of black additive (subtractive) secrets that the leaving user has. It is likely that $n_{1} \neq\left|\mathcal{S}_{1}\right|\left(n_{2} \neq\left|\mathcal{S}_{2}\right|\right)$. The key dealer runs the following procedure.

- It finds the smallest $x^{\prime}$ such that $\left(\begin{array}{c}n_{1}+x^{\prime} \\ x^{\prime}\end{array}\right)\left(\begin{array}{c}n_{2}+x^{\prime} \\ x^{\prime}\end{array}\right) \geq 2^{l}$.

- It randomly selects $\varphi$ existing users as helper users, where $\varphi$ is large enough to ensure that, given $\gamma$, at least one helper user is good. If any helper user has less than $x^{\prime}+x$ black additive (subtractive) secrets, the key dealer runs the setup phase again.

- It removes $x^{\prime}$ black additive (subtractive) secrets from each helper user and these secrets form a set $\mathcal{S}_{3}\left(\mathcal{S}_{4}\right)$.

- It evenly and randomly redistributes the black additive (subtractive) secrets in $\mathcal{S}_{1} \cup \mathcal{S}_{3}\left(\mathcal{S}_{2} \cup \mathcal{S}_{4}\right)$ to the $\varphi$ helper users.

- It tags all the redistributed secrets as white.

- It counts the minimum possible total number of black additive (subtractive) secrets, similarly as done in the join operation. If the number is smaller than $b$, it reruns the setup phase.

If the leaving user did not participate in the previous setup phase (i.e., it joins the system after the previous setup phase), the key dealer does not know the accurate value of $n_{1}$ and $n_{2}$. However, it knows that $n_{1} \geq x$ and $n_{2} \geq x$. To be conservative, it sets $n_{1}=x$ and $n_{2}=x$ in this case.

Security analysis. In the worst case, the adversary may identify the $n_{1}+x^{\prime}$ additive (subtractive) secrets that belong to the leaving user and one good helper user. However, since $\left(\begin{array}{c}n_{1}+x^{\prime} \\ x^{\prime}\end{array}\right)\left(\begin{array}{c}n_{2}+x^{\prime} \\ x^{\prime}\end{array}\right) \geq 2^{l}$, the leaving user's backward security is guaranteed. Since each other user has at least $x$ black additive (subtractive) secrets and the total number of black additive (subtractive) secrets is not smaller than $b$ after the leave operation, the forward security of other users is satisfied. Each user's backward security is also guaranteed, since there are more black secrets before the leave operation.

\section{Practical Considerations}

Given $n$ and $\gamma$, the values of parameters $\varphi, b, x$, and $c$ can be set as follows.

1) Setting $\varphi$ : Given $\gamma, \varphi$ should be large enough such that at least one helper user is good. If we assume that the colluding users are a random subset of the user set, the value of $\varphi$ should be sufficiently large such that with a probability (denoted by $p_{s}$ ) not lower than $1-2^{-l}$ at least one helper user is good. Under this random colluder model, we can derive that $p_{s}=$ $1-\gamma^{\varphi}$. Thus,

$$
\varphi=\left\lceil-l \log _{\gamma} 2\right\rceil
$$


TABLE XI

THE VALUES OF $\varphi$ FOR 80 -BIT SECURITY UNDER THE RANDOM COLLUDER MODEL.

\begin{tabular}{ccccccc}
\hline$\gamma$ & 0 & 0.01 & 0.05 & 0.1 & 0.15 & 0.2 \\
\hline$\varphi$ & 1 & 13 & 19 & 25 & 30 & 35 \\
\hline
\end{tabular}

TABLE XII

THE VALUES OF $b, x$ AND $q$ USED FOR DEALING WITH DYNAMIC JOINS AND LEAVES WHEN THE SECURITY LEVEL IS 80-BIT.

\begin{tabular}{cccccc}
\hline$n$ & $10^{2}$ & $10^{3}$ & $10^{4}$ & $10^{5}$ & $10^{6}$ \\
\hline$x$ & 6 & 4 & 3 & 3 & 2 \\
\hline$b$ & 600 & 4000 & $3 \cdot 10^{4}$ & $3 \cdot 10^{5}$ & $2 \cdot 10^{6}$ \\
\hline$q$ & 12 & 8 & 7 & 5 & 5 \\
\hline
\end{tabular}

Table XI shows the values of $\varphi$ when $l=80$.

2) Setting $b, x$ and $c$ : For simplicity, we can set $b=n x$, and then derive the value of $x$ which is the minimum integer that satisfies Equation 16. Then the value of $b$ can also be obtained as the value of $x$ multiplied by $n$. From $b$, we can derive the value of $q$ which is the minimum integer that satisfies Equation 17. Table XII show the parameter values obtained in this way.

Then we can set $c=K x$ (e.g., $K=10$ ). Parameter $K$ is a tuning factor which affects both the number of joins and leaves supported without rerunning the setup phase and the computation overhead of at each user. When $K$ increases, more joins and leaves can be supported without rerunning the setup phase; however, since $c$ is larger, the communication overhead at user is also higher. We will evaluate the effect of $K$ in Section VII.

\section{Evaluations}

This section evaluates the cost of our aggregation protocols for Sum and Min. We compare our solution against three existing privacy-preserving aggregation protocols for timeseries data: the protocol proposed in [7] (denoted by EXP), CollaPSE [9], and the spatial aggregation protocol proposed in [24] (denoted by Spatial).

\section{A. The Cost of Sum and Min Aggregation}

This section compares our Sum and Min aggregation protocols (see Section IV and V-B) against existing work.

1) Cost of Sum aggregation: EXP is a Sum aggregation protocol based on the decisional Diffie-Hellman assumption. In EXP, encryption (decryption) requires two $(\sqrt{n \Delta})$ modular exponentiations (see [7] for details). Similar to our protocol, CollaPSE also uses the homomorphic encryption scheme in [20] to derive Sum, but in a different way (see [9] for details). In CollaPSE, each user (the aggregator) computes $s+1$ PRFs to encrypt her data (decrypt the sum). Here, $s$ denotes the number of colluding users that the protocol can tolerate. Spatial is based on the Paillier cryptosystem.

Table XIII shows the computation, storage, and communication cost of the four aggregation protocols for Sum, where the cost is derived under the same condition that they can tolerate $\gamma n$ colluding users. Compared with CollaPSE, our protocol has much smaller computation and storage cost at both the users and the aggregator, especially for a large system with possibly many colluding users. Compared with EXP and Spatial, our protocol has slightly higher storage cost (i.e., around 10 secrets each with just tens of bytes), but our computation overhead is much lower since in practice PRF (when implemented with HMAC) can run orders of magnitude faster than modular exponentiation, Paillier encryption, and Paillier decryption. We elaborate this point further in Section VII-A3. Our protocol also has much lower communication cost than Spatial.

2) Cost of Min aggregation: The Min aggregation scheme presented in Section V-B derives Min from $2^{\epsilon-1}(\log \Delta+2)$ parallel Sum aggregates of 1-bit data, where each sum is obtained using our Sum aggregation protocol. Here, each sum can also be obtained using EXP, and we refer to the Min aggregation scheme that uses EXP as a building block as EXPMin. In EXP-Min, each user computes $2^{\epsilon}(\log \Delta+2)$ modular exponentiations to encrypt her data, and the aggregator computes $2^{\epsilon-1} \sqrt{n}(\log \Delta+2)$ modular exponentiations to decrypt the Min. Similarly, CollaPSE can also be used as a building block of Min aggregation, and the resulting scheme is referred to as CollaPSE-Min. In CollaPSE-Min, the computation cost is $\frac{2^{\epsilon-1}(\gamma n+1)(\log \Delta+2)}{H}$ PRFs for both encryption and decryption, considering that the concatenation technique in Section V-A also works for CollaPSE.

Compared with CollaPSE-Min, our Min aggregation scheme improves the computation cost of encryption and decryption by a factor of $\frac{\gamma n+1}{2 c}$ and $\frac{\gamma n+1}{q}$ respectively (see Table IX for the cost of our scheme). Our scheme is also much more efficient than EXP-Min in computation, as shown in Section VII-A3.

3) Practical Performances: In Table XIII, the computation costs of our Sum aggregation protocol, EXP, and Spatial are measured by different units. Here, we elaborate the comparison between them with results in running time. For this purpose, we implemented these protocols in Java. The function of mobile user is implemented on Nexus S Smartphone with Android 4.0.4 OS, 1GHz CPU, and 512MB RAM. The function of aggregator is implemented on a Windows Laptop with 64-bit Windows $7 \mathrm{OS}, 2.6 \mathrm{GHz} \mathrm{CPU}$, and 4GB RAM. For EXP, the elliptic curve "curve25519" is adopted for modular exponentiation. For Spatial, the Paillier cryptosystem uses a 1024-bit modulus, and random numbers are generated using the standard function provided by Java. For our protocol, PRF is implemented with HMAC-SHA256.

Table XIV shows the running time of our Sum aggregation protocol, EXP, and Spatial. Our protocol is much faster than EXP and Spatial in both encryption and decryption. Specifically, encryption is at least one order of magnitude faster. When the plaintext space $\Delta \geq 10^{2}$, decryption is at least three orders of magnitudes faster. In our protocol, the computation cost decreases as the system scale increases, and it does not change with the plaintext space (so long as the size of plaintext data does not exceed the size of an HMAC output). Thus, our protocol can support large systems and large plaintext spaces.

Table XV shows the running time of our Min aggregation protocol and EXP-Min. Here, the plaintext space is set as $\Delta=$ $10^{4}$. The parameters of our protocol are set according to Table $\mathrm{V}$ and Table VI when $\gamma=0.2$. In all the shown cases, our protocol is at least four (seven) orders of magnitude faster than EXP-Min in encryption (decryption). Especially, as the system 
TABLE XIII

COMPARISONS BETWEEN OUR SUM AGGREGATION PROTOCOL AND EXISTING PROTOCOLS.

\begin{tabular}{|c|c|c|c|c|c|}
\hline & Encryption (user) & Decryption (aggregator) & Storage (user) & Storage (aggr.) & Communication \\
\hline EXP & 2 Mod. Exp. & $\sqrt{n \Delta}$ Mod. Exp. & 1 & 1 & $\mathrm{O}(n)$ \\
\hline CollaPSE & $(\gamma n+1)$ PRFs & $(\gamma n+1)$ PRFs & $\gamma n+1$ & $\gamma n+1$ & $\mathrm{O}(n)$ \\
\hline Spatial & 1 Paillier Enc. $+(n-1)$ PRFs & 1 Paillier Dec. $+(n-1)$ Mod. Mul. & 1 & 1 & $\mathrm{O}\left(n^{2}\right)$ \\
\hline Ours & $2 c$ PRFs & $q$ PRFs & $2 c$ & $q$ & $\mathrm{O}(n)$ \\
\hline
\end{tabular}

Mod. Exp. (Mul.) stands for modular exponentiations (multiplications). In most practical settings, $c \leq 7$ and $q \leq 13$ (see Table V and VI).

TABLE XIV

THE RUNNING TIME OF OUR SUM PROTOCOL, EXP, AND SPATIAL.

\begin{tabular}{|c|c|ccccc|}
\hline & $n$ & $10^{2}$ & $10^{3}$ & $10^{4}$ & $10^{5}$ & $10^{6}$ \\
\hline \hline Enc. & Ours & $2.9 \mathrm{~ms}$ & $2.4 \mathrm{~ms}$ & $2.1 \mathrm{~ms}$ & $1.9 \mathrm{~ms}$ & $1.4 \mathrm{~ms}$ \\
& Spatial & $115 \mathrm{~ms}$ & $140 \mathrm{~ms}$ & $392 \mathrm{~ms}$ & $2.9 \mathrm{~s}$ & $28 \mathrm{~s}$ \\
& EXP & $90 \mathrm{~ms}$ & $90 \mathrm{~ms}$ & $90 \mathrm{~ms}$ & $90 \mathrm{~ms}$ & $90 \mathrm{~ms}$ \\
\hline Dec. & Ours & $39 \mu \mathrm{s}$ & $24 \mu \mathrm{s}$ & $18 \mu \mathrm{s}$ & $15 \mu \mathrm{s}$ & $12 \mu \mathrm{s}$ \\
& Spatial & $15 \mathrm{~ms}$ & $38 \mathrm{~ms}$ & $263 \mathrm{~ms}$ & $2.5 \mathrm{~s}$ & $25 \mathrm{~s}$ \\
& $\operatorname{EXP}\left(\Delta=10^{2}\right)$ & $560 \mathrm{~ms}$ & $1.8 \mathrm{~s}$ & $5.6 \mathrm{~s}$ & $18 \mathrm{~s}$ & $56 \mathrm{~s}$ \\
& $\operatorname{EXP}\left(\Delta=10^{3}\right)$ & $1.8 \mathrm{~s}$ & $5.6 \mathrm{~s}$ & $18 \mathrm{~s}$ & $56 \mathrm{~s}$ & $177 \mathrm{~s}$ \\
& $\operatorname{EXP}\left(\Delta=10^{4}\right)$ & $5.6 \mathrm{~s}$ & $18 \mathrm{~s}$ & $56 \mathrm{~s}$ & $177 \mathrm{~s}$ & $560 \mathrm{~s}$ \\
& $\operatorname{EXP}\left(\Delta=10^{5}\right)$ & $18 \mathrm{~s}$ & $56 \mathrm{~s}$ & $177 \mathrm{~s}$ & $560 \mathrm{~s}$ & $1770 \mathrm{~s}$ \\
\hline
\end{tabular}

$\Delta$ is the plaintext space. Each user's data value is from $\{0,1, \ldots, \Delta\}$.

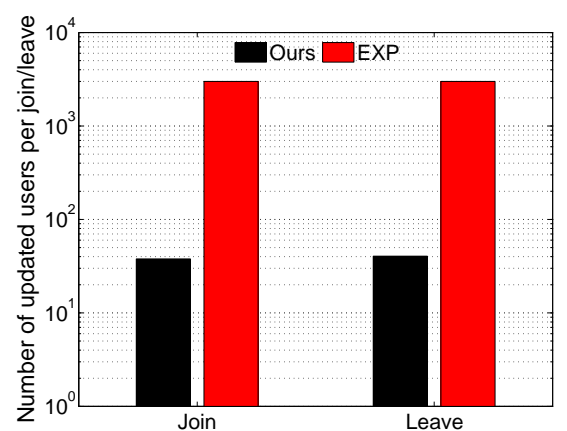

Fig. 7. Comparisons with EXP in the average number of updated users per join and leave. Please note the logarithmic scale.

scale increases, the running time of decryption in EXP-Min increases quickly which shows the poor scalability of EXPMin, but the running time of our protocol decreases and is always very low, which shows that our protocol is scalable.

\section{B. The Cost of Dynamic Joins and Leaves}

This section evaluates our scheme for dealing with joins and leaves (see Section VI). Each data point is the average result of ten simulation runs with different random seeds.

1) The effect of $K, n$ and $\gamma$ : In our scheme, after the setup phase, a number of joins and leaves can be processed before the setup phase has to be run again. We first run simulations to evaluate the effect of parameters $K$ (see Section VI-D2), $n$ and $\gamma$ on this number.

Figure 6 shows the results. As can be seen from Figure 6(a), when $K$ increases, more joins and leaves can be supported (where only $\varphi$ users are updated) without rerunning the setup phase again. This is because when $K$ is larger there is more redundancy in security and hence the communication cost is lower. For similar reasons, when $n$ increases, more joins and leaves can also be supported (see Figure 6(b)). When $\gamma$ increases, the setup phase needs to be run again sooner (see Figure 6(c)). The reason is that when $\gamma$ is higher, $\varphi$ is larger, which means more users are updated and more secrets are transited from black to white in each join.
TABLE XVI

THE RUNNING TIME OF OUR PROTOCOL FOR SUM WITH REDUNDANCY IN SECURITY AND THE RUNNING TIME OF EXP.

\begin{tabular}{|c|c|ccccc|}
\hline & $n$ & $10^{2}$ & $10^{3}$ & $10^{4}$ & $10^{5}$ & $10^{6}$ \\
\hline \hline Enc. & Ours & $144 \mathrm{~ms}$ & $96 \mathrm{~ms}$ & $72 \mathrm{~ms}$ & $72 \mathrm{~ms}$ & $48 \mathrm{~ms}$ \\
& EXP & $90 \mathrm{~ms}$ & $90 \mathrm{~ms}$ & $90 \mathrm{~ms}$ & $90 \mathrm{~ms}$ & $90 \mathrm{~ms}$ \\
\hline Dec. & Ours & $36 \mu \mathrm{s}$ & $24 \mu \mathrm{s}$ & $21 \mu \mathrm{s}$ & $15 \mu \mathrm{s}$ & $15 \mu \mathrm{s}$ \\
& $\operatorname{EXP}\left(\Delta=10^{2}\right)$ & $560 \mathrm{~ms}$ & $1.8 \mathrm{~s}$ & $5.6 \mathrm{~s}$ & $18 \mathrm{~s}$ & $56 \mathrm{~s}$ \\
& $\operatorname{EXP}\left(\Delta=10^{3}\right)$ & $1.8 \mathrm{~s}$ & $5.6 \mathrm{~s}$ & $18 \mathrm{~s}$ & $56 \mathrm{~s}$ & $177 \mathrm{~s}$ \\
& $\operatorname{EXP}\left(\Delta=10^{4}\right)$ & $5.6 \mathrm{~s}$ & $18 \mathrm{~s}$ & $56 \mathrm{~s}$ & $177 \mathrm{~s}$ & $560 \mathrm{~s}$ \\
& $\operatorname{EXP}\left(\Delta=10^{5}\right)$ & $18 \mathrm{~s}$ & $56 \mathrm{~s}$ & $177 \mathrm{~s}$ & $560 \mathrm{~s}$ & $1770 \mathrm{~s}$ \\
\hline
\end{tabular}

TABLE XVII

THE STORAGE COST OF OUR PROTOCOL FOR SUM WITH REDUNDANCY IN SECURITY. $K=50 . \gamma=0.2$.

\begin{tabular}{|c|ccccc|}
\hline$n$ & $10^{2}$ & $10^{3}$ & $10^{4}$ & $10^{5}$ & $10^{6}$ \\
\hline Num. of secrets (user) & 600 & 500 & 400 & 300 & 300 \\
\hline Num. of secrets (aggr.) & 13 & 8 & 6 & 5 & 4 \\
\hline
\end{tabular}

The computation and storage overhead at mobile user linearly increases with $K$. Thus, in practice, the value of $K$ should be selected based on the estimated $\gamma$, the expected level of churn resilience, and the tolerable computation and storage cost. In the following, we set $K=50$.

2) Cost: Then we compare the communication cost of our scheme against EXP in dealing with dynamic joins and leaves. In the simulations for join (leave), initially there are 1000 (5000) users in the system and 4000 users join (leave) subsequently. We set $K=50$ and $\gamma=0.2$. Figure 7 shows the average number of updated users per join and leave. Clearly, our scheme has much lower communication cost. This is because EXP redistributes secrets to all users upon every join and leave, but most of the time our scheme redistributes secrets to a small subset of users.

The computation cost of our scheme is increased when dealing with dynamic joins and leaves. To evaluate the computation cost, we measured the running time when there is redundancy in security. Here, the parameters of our scheme are set as discussed in Section VI-D and $K=50$. As shown in Table XVI, the running time of encryption in our scheme is comparable to that in EXP, but the running time of decryption in our scheme is still orders of magnitude faster.

The storage cost at each mobile user is also increased. However, as shown in Table XVII, each user only stores hundreds of secrets. Considering that each secret has only tens of bytes, the storage cost is low.

\section{DISCUSSIONS}

Relaxing the assumption of trusted key dealer Instead of relying on a trusted key dealer, our protocol can be easily adapted to work with an honest-but-curious key dealer that does not collude with the aggregator. An honest-but-curious 
TABLE XV

THE RUNNING TIME OF OUR MIN AGgREGATION PROTOCOL AND EXP-MIN WHICH USES EXP AS A BUILDING BLOCK.

\begin{tabular}{|c|c|c|c|c|c|c|c|c|c|}
\hline & \multicolumn{4}{|c|}{ Encryption } & \multicolumn{4}{|c|}{ Decryption } \\
\hline & & $n=10^{3}$ & $n=10^{4}$ & $n=10^{5}$ & $n=10^{6}$ & $n=10^{3}$ & $n=10^{4}$ & $n=10^{5}$ & $n=10^{6}$ \\
\hline \multirow{2}{*}{ Relative error $<1 \%$} & EXP-Min & $88 \mathrm{~s}$ & $88 \mathrm{~s}$ & $88 \mathrm{~s}$ & $88 \mathrm{~s}$ & $173 \mathrm{~s}$ & $548 s$ & $1733 \mathrm{~s}$ & $5479 s$ \\
\hline & Ours & $9.2 \mathrm{~ms}$ & $7.3 \mathrm{~ms}$ & $5.5 \mathrm{~ms}$ & $5.5 \mathrm{~ms}$ & $92 \mu \mathrm{s}$ & $69 \mu \mathrm{s}$ & $57 \mu \mathrm{s}$ & $46 \mu \mathrm{s}$ \\
\hline \multirow{2}{*}{ Relative error $<0.1 \%$} & EXP-Min & $704 s$ & $704 s$ & $704 \mathrm{~s}$ & $704 s$ & $1386 \mathrm{~s}$ & 4383 & $13861 \mathrm{~s}$ & $43833 s$ \\
\hline & Ours & $73 \mathrm{~ms}$ & $59 \mathrm{~ms}$ & $44 \mathrm{~ms}$ & $44 \mathrm{~ms}$ & $734 \mu \mathrm{s}$ & $550 \mu \mathrm{s}$ & $459 \mu \mathrm{s}$ & $367 \mu \mathrm{s}$ \\
\hline
\end{tabular}

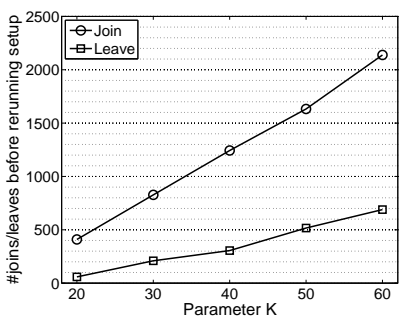

(a) Parameter $K$

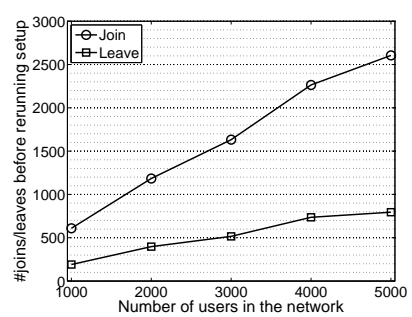

(b) Parameter $n$

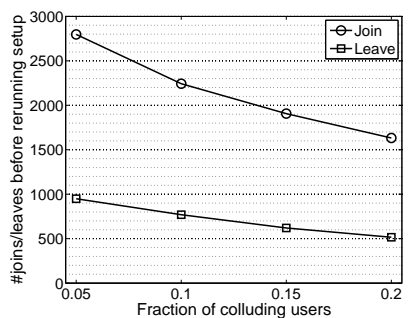

(c) Parameter $\gamma$

Fig. 6. The effects of parameters $K$ (see Section VI-D2), $n$ and $\gamma$ on the number of joins/leaves supported by each setup phase. By default, $K=50$, $\gamma=0.2$, and $n=3000$ (i.e., the number of users at the beginning of simulation).

key dealer correctly follows our protocol steps, but wants to get users' data values from the transcript of messages in our protocol. To provide privacy under this model, our protocol adds one more encryption and decryption to the data that each user submits to the aggregator. More specifically, each user encrypts its data using the secrets assigned by the key dealer to derive an intermediate result $z$, encrypts $z$ with a key pre-shared with the aggregator, and then sends the obtained ciphertext to the aggregator. The aggregator first uses the preshared key to decrypt each user's intermediate result $z$, and then decrypts the noisy sum with the secrets received from the key dealer. The key dealer cannot obtain the intermediate result $z$ of any user, so long as it does not collude with the aggregator. Hence, it cannot get any user's data value.

The honest-but-curious model is realistic because it can be enforced with trusted hardware. In practice, certificate authorities such as VeriSign (which already provides key management services) may serve as the key dealer. Since these authorities usually undergo extensive audits (e.g., WebTrust audit), collusion with the aggregator can be mitigated.

More aggregate statistics. In the basic aggregation scheme for Min presented in Section V-A, the aggregator can actually get the number of times that each possible data value appears, and derive the accurate distribution of the users' data in the plaintext space $[0, \Delta]$. From the distribution, other aggregate statistics such as Median, Percentile and Histogram can be obtained. In this process, the aggregator knows nothing about each individual user's data.

Differential privacy for Sum. Differential privacy [31] provides strong privacy guarantee for users such that a user's participation in the system only leaks negligible information about the user. Our protocol for Sum can be adapted to provide computational differential privacy [8]. To achieve this goal, an appropriate noise is added to each user's data (e.g., using the data perturbation algorithms proposed in [7]), and then the sum of noisy data is obtained using our protocol.

Fault tolerance Fault tolerance requires that when a number of users fail (e.g., due to loss of power or network connection), the aggregator can still get the aggregate statistics of the remaining users. There is a collision between aggregator obliviousness and fault tolerance. Data perturbation [7] can be used to address such collision. With data perturbation, the binary construction proposed in [8] can be applied on top of our Sum aggregation protocol to achieve fault tolerance.

Weaker assumption on colluders In previous sections, we assumed that $\gamma$ is the maximum accumulated fraction of colluding users in the lifetime of the system. Since the system lifetime may be very long, $\gamma$ may be large (i.e., close to 1) which makes the value of parameter $c$ large. However, this assumption can be relaxed as follows. The key dealer can rerun the setup phase in every time period $T$ (e.g., one month) and issue a new set of secrets. Then $\gamma$ can be the maximum accumulated fraction of colluding users in time period $T$.

\section{CONClusions}

To facilitate the collection of useful aggregate statistics in mobile sensing without leaking mobile users' privacy, we proposed a new privacy-preserving protocol to obtain the Sum aggregate of time-series data. The protocol utilizes additive homomorphic encryption and a novel, HMAC-based key management technique to perform extremely efficient aggregation. Implementation-based measurements show that operations at user and aggregator in our protocol are orders of magnitude faster than existing work. Thus, our protocol can be applied to a wide range of mobile sensing systems with various scales, plaintext spaces, aggregation loads and resource constraints.

Based on the Sum aggregation protocol, we also proposed two schemes to derive the Min aggregate of time-series data. One scheme can obtain the accurate Min while the other one can obtain an approximate Min with provable error guarantee at much lower cost.

To deal with dynamic joins and leaves, we proposed a scheme which utilizes the redundancy in security to reduce the communication cost for each join and leave. Simulation 
results show that our scheme has much lower communication overhead than existing work.

\section{ACKNOWLEDGMENT}

The authors would like to thank the anonymous reviewers for their insightful comments. A preliminary version [32] of this paper appeared in IEEE ICNP 2012.

\section{REFERENCES}

[1] M. Mun, S. Reddy, K. Shilton, N. Yau, J. Burke, D. Estrin, M. Hansen, E. Howard, R. West, and P. Boda, "Peir, the personal environmental impact report, as a platform for participatory sensing systems research," in Proc. ACM MobiSys, 2009, pp. 55-68.

[2] A. Thiagarajan, L. Ravindranath, K. LaCurts, S. Madden, H. Balakrishnan, S. Toledo, and J. Eriksson, "Vtrack: accurate, energy-aware road traffic delay estimation using mobile phones," in Proc. ACM SenSys, 2009, pp. 85-98.

[3] S. Consolvo, D. W. McDonald, T. Toscos, M. Y. Chen, J. Froehlich, B. Harrison, P. Klasnja, A. LaMarca, L. LeGrand, R. Libby, I. Smith, and J. A. Landay, "Activity sensing in the wild: a field trial of ubifit garden," in Proc. CHI, 2008, pp. 1797-1806.

[4] J. Hicks, N. Ramanathan, D. Kim, M. Monibi, J. Selsky, M. Hansen, and D. Estrin, "Andwellness: an open mobile system for activity and experience sampling," in Proc. Wireless Health, 2010, pp. 34-43.

[5] N. D. Lane, M. Mohammod, M. Lin, X. Yang, H. Lu, S. Ali, A. Doryab, E. Berke, T. Choudhury, and A. Campbell, "Bewell: A smartphone application to monitor, model and promote wellbeing," in 5th Int'l ICST Conf. on Pervasive Computing Technologies for Healthcare, 2011.

[6] V. Rastogi and S. Nath, "Differentially private aggregation of distributed time-series with transformation and encryption," ACM SIGMOD, 2010.

[7] E. Shi, T.-H. H. Chan, E. Rieffel, R. Chow, and D. Song, "Privacypreserving aggregation of time-series data," Network and Distributed System Security Symposium (NDSS), 2011.

[8] T.-H. H. Chan, E. Shi, and D. Song, "Privacy-preserving stream aggregation with fault tolerance," Financial Cryptography and Data Security $(F C), 2012$.

[9] E. G. Rieffel, J. Biehl, W. van Melle, and A. J. Lee, "Secured histories: computing group statistics on encrypted data while preserving individual privacy." In submission, 2010.

[10] P.-A. Fouque, G. Poupard, and J. Stern, "Sharing decryption in the context of voting or lotteries," in Proceedings of the 4th International Conference on Financial Cryptography, ser. FC '00, 2000, pp. 90-104.

[11] MNDOLI, "Mnosha permissible exposure limits," available at http://www.dli.mn.gov/OSHA/PDF/pels.pdf.

[12] S. B. Eisenman, E. Miluzzo, N. D. Lane, R. A. Peterson, G.-S. Ahn, and A. T. Campbell, "The bikenet mobile sensing system for cyclist experience mapping," in Proc. ACM SenSys, 2007, pp. 87-101.

[13] M. G. Apte, W. J. Fisk, and J. M. Daisey, "Indoor carbon dioxide concentrations and sbs in office workers," in Proceedings of Healthy Buildings, 2000, pp. 133-138.

[14] Q. Li and G. Cao, "Providing privacy-aware incentives for mobile sensing," in Proc. IEEE PerCom, 2013.

[15] Z. Zhu and G. Cao, "Applaus: A privacy-preserving location proo updating system for location-based services," in IEEE INFOCOM, 2011.

[16] Q. Li and G. Cao, "Mitigating routing misbehavior in disruption toleran networks," IEEE Transactions on Information Forensics and Security, vol. 7, no. 2, pp. 664-675, April 2012.

[17] E. D. Cristofaro and C. Soriente, "Short paper: Pepsi-privacy-enhanced participatory sensing infrastructure," in Proc. ACM WiSec, 2011.

[18] Q. Li, W. Gao, S. Zhu, and G. Cao, "To lie or to comply: Defending against flood attacks in disruption tolerant networks," IEEE Trans. on Dependable and Secure Computing, vol. 10, no. 3, pp. 168-182, 2013.

[19] C. Gentry, "Fully homomorphic encryption using ideal lattices," in ACM symposium on Theory of computing (STOC), 2009, pp. 169-178.

[20] C. Castelluccia, A. C.-F. Chan, E. Mykletun, and G. Tsudik, "Efficient and provably secure aggregation of encrypted data in wireless sensor networks," ACM Transactions on Sensor Networks (TOSN), vol. 5, no. 3, pp. 20:1-20:36, 2009

[21] Y. Yang, X. Wang, S. Zhu, and G. Cao, "Sdap: A secure hop-by-hop data aggregation protocol for sensor networks," ACM Transactions on Information and System Security (TISSEC), vol. 11, no. 4, 2008.
[22] Z. Yang, S. Zhong, and R. N. Wright, "Privacy-preserving classification of customer data without loss of accuracy," in SIAM SDM, 2005.

[23] J. Shi, R. Zhang, Y. Liu, and Y. Zhang, "Prisense: privacy-preserving data aggregation in people-centric urban sensing systems," in Proc. IEEE INFOCOM, 2010, pp. 758-766.

[24] Z. Erkin and G. Tsudik, "Private computation of spatial and temporal power consumption with smart meters," in Proc. ACNS, 2012.

[25] G. Ács and C. Castelluccia, "I have a dream!: differentially private smart metering," in Int'l conf. on Information hiding, 2011, pp. 118-132.

[26] M. Jawurek and F. Kerschbaum, "Fault-tolerant privacy-preserving statistics," in Privacy Enhancing Technologies Symposium (PETS), 2012

[27] Q. Li and G. Cao, "Efficient privacy-preserving stream aggregation in mobile sensing with low aggregation error,' Privacy Enhancing Technologies Symposium (PETS), 2013.

[28] M. Shao, Y. Yang, S. Zhu, and G. Cao, "Towards statistically strong source anonymity for sensor networks," in Proc. IEEE INFOCOM, 2008.

[29] C. Castelluccia, "Efficient aggregation of encrypted data in wireless sensor networks," in MobiQuitous, 2005, pp. 109-117.

[30] M. Bellare, "New proofs for nmac and hmac: security without collisionresistance," in Proc. CRYPTO, 2006, pp. 602-619.

[31] C. Dwork, F. McSherry, K. Nissim, and A. Smith, "Calibrating noise to sensitivity in private data analysis," TCC, 2006.

[32] Q. Li and G. Cao, "Efficient and privacy-preserving data aggregation in mobile sensing," in Proc. IEEE ICNP, 2012, pp. 1-10.

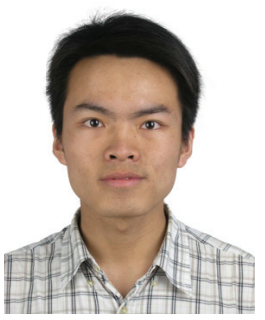

Qinghua Li received the B.E. degree from Xian Jiaotong University, China, and the M.S. degree from Tsinghua University, China, in 2004 and 2007, respectively. He is currently a Ph.D. candidate in the Department of Computer Science and Engineering at the Pennsylvania State University. His research interests include security and privacy in networking systems. He is a student member of the IEEE.

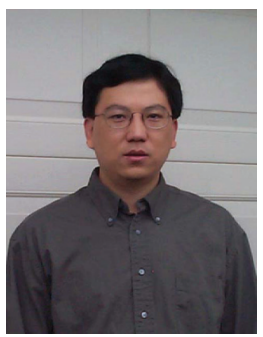

Guohong Cao received the BS degree in computer science from Xian Jiaotong University and received the $\mathrm{PhD}$ degree in computer science from the Ohio State University in 1999. Since then, he has been with the Department of Computer Science and Engineering at the Pennsylvania State University, where he is currently a Professor. He has published more than 200 papers in the areas of wireless networks, wireless security, vehicular networks, wireless sensor networks, cache management, and distributed fault tolerant computing. He has served on the editorial board of IEEE Transactions on Mobile Computing, IEEE Transactions on Wireless Communications, IEEE Transactions on Vehicular Technology, and has served on the organizing and technical program committees of many conferences, including the TPC Chair/Co-Chair of IEEE SRDS'2009, MASS'2010, and INFOCOM'2013. He was a recipient of the NSF CAREER award in 2001. He is a Fellow of the IEEE.

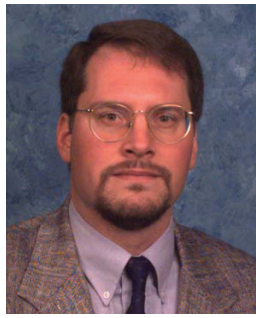

Thomas F. La Porta is the William E. Leonhard Chair Professor in the Computer Science and Engineering Department at Penn State. He received his B.S.E.E. and M.S.E.E. degrees from The Cooper Union, New York, NY, and his Ph.D. degree in Electrical Engineering from Columbia University, New York, NY. He joined Penn State in 2002. $\mathrm{He}$ is the Director of the Networking and Security Research Center at Penn State. Prior to joining Penn State, Dr. La Porta was the Director of the Mobile Networking Research Department in Bell Laboratories. He is an IEEE Fellow and Bell Labs Fellow. Dr. La Porta was the founding Editor-in-Chief of the IEEE Transactions on Mobile Computing, and currently serves on its Steering Committee. He was the Director of Magazines for the IEEE Communications Society and was on its Board of Governors for three years. He has published numerous papers and holds 35 patents. His research interests include mobility management, signaling and control for wireless networks, security for wireless systems, mobile data systems, and protocol design. 